\title{
ARTICLE Reward sensitivity deficits in a rat model of compulsive eating behavior
}

\author{
Catherine F. Moore ${ }^{1,2}$, Michael Z. Leonard ${ }^{3}$, Nicholas M. Micovic ${ }^{1}$, Klaus A. Miczek ${ }^{3,4}$, Valentina Sabino ${ }^{1}$ and Pietro Cottone ${ }^{1}$
}

Compulsive eating behavior is hypothesized to be driven in part by reward deficits likely due to neuroadaptations to the mesolimbic dopamine (DA) system. Therefore, the aim of this study was to assess deficits in reward system functioning and mesolimbic DA after alternating a standard chow with palatable diet, a model of compulsive eating. In this model, rats in the control group (Chow/Chow) are provided a standard chow diet 7 days a week, while the experimental group (Chow/Palatable) is provided chow for 5 days a week ("C Phase"), followed by 2 days of access to a highly palatable sucrose diet ("P Phase"). We first tested the sensitivity to $d$-Amphetamine's stimulatory, reward-enhancing, and primary rewarding effects using a locomotor activity assay, an intracranial self-stimulation (ICSS) procedure, and a conditioned place preference test, respectively. We then quantified DA release in the nucleus accumbens (NAc) shell after treatment with $d$-Amphetamine using in vivo microdialysis, quantified levels of tyrosine hydroxylase (TH) and dopamine transporter (DAT) mRNA using quantitative polymerase chain reaction (qPCR), and lastly, quantified baseline extracellular DA and function of DAT in vivo using quantitative "no-net-flux" microdialysis. Chow/Palatable rats displayed blunted $d$-Amphetamine-induced locomotor activity, insensitivity to $d$-Amphetamine potentiation of ICSS threshold, and decreased place preference for $d$-Amphetamine during the P Phase. We found that Chow/Palatable rats had blunted DA efflux following $d$-Amphetamine treatment. Furthermore, DAT mRNA was increased in Chow/Palatable rats during the P Phase. Finally, quantitative "no-net-flux" microdialysis revealed reduced extracellular baseline DA and DAT function in Chow/Palatable rats. Altogether, these results provide evidence of reduced reward system functioning and related neuroadaptations in the DA and DAT systems in this model of compulsive eating. Reward deficits, resulting from repeated overeating, may in turn contribute to the perpetuation of compulsive eating behavior.

Neuropsychopharmacology (2020) 45:589-596; https://doi.org/10.1038/s41386-019-0550-1

\section{INTRODUCTION}

Obesity and eating disorders are characterized by compulsive eating behavior, an emerging construct similar to compulsive drug use in substance use disorders [1]. Compulsive eating behavior is complex and multifaceted: overeating to relieve a negative emotional state is considered to be a key element of compulsive eating [1-3], rooted in evidence from both preclinical and clinical research $[3,4]$. Overeating to relieve a negative emotional state is thought to be driven by two processes: diminished reward sensitivity and the emergence of negative affect $[1,5,6]$.

Similar to drug use, consumption of palatable food is hypothesized to result in repeated stimulation, and ultimately in desensitization, of the mesolimbic dopamine (DA) system, a major substrate of reward and reinforcement [7-9]. Continued overeating may therefore reflect the need to reactivate a hypofunctional reward circuit $[10,11]$. Animal models of obesity and binge eating have observed downregulated DA signaling, lower DA turnover, and reduced activity of the dopamine transporter (DAT), which regulates synaptic concentrations of DA via reuptake [10, 12-14].

Preclinically, one way to model compulsive eating behavior is to implement an intermittent access schedule to a palatable diet, consisting of alternating access to a highly palatable, high-sucrose, chocolate-flavored diet (preferred) with a lesser preferred, standard chow diet [4, 15]. This model has been shown to comprise core features of compulsive eating behavior in rats [16], namely, overeating despite negative consequences [15] as well as overeating to alleviate a negative emotional state $[1,17]$. This model is especially relevant, as all food is given without food restriction and rats do not become obese, therefore decoupling any effects of overeating from an overweight/obese phenotype $[15,17,18]$. Furthermore, this model allows us to investigate any dissociable effects of present feeding state (current palatable food exposure vs. withdrawal) on reward sensitivity deficits.

Therefore, the first aim of this series of experiments was to assess deficits in reward system functioning in the aforementioned animal model of compulsive eating. For this purpose, we tested sensitivity to $d$-Amphetamine, a drug which acts by inhibiting DA reuptake as well as promoting its release, in rats with a history of alternating a standard chow with palatable diet. Specifically, we tested response to $d$-Amphetamine's stimulatory, reward enhancing, and primary rewarding effects (using a locomotor activity assay [19], an intracranial self-stimulation (ICSS) procedure [20], and a conditioned place preference (CPP) test [21], respectively). We also directly quantified DA release in the nucleus

\footnotetext{
${ }^{1}$ Laboratory of Addictive Disorders, Departments of Pharmacology and Psychiatry, Boston University School of Medicine, Boston, MA, USA; ${ }^{2}$ Graduate Program for Neuroscience, Boston University School of Medicine, Boston, MA, USA; ${ }^{3}$ Department of Psychology, Tufts University, Medford, MA, USA and ${ }^{4}$ Departments of Neuroscience, Psychiatry and Pharmacology, Tufts University, Boston, MA, USA

Correspondence: Pietro Cottone (cottone@bu.edu)
}

Received: 29 July 2019 Revised: 7 October 2019 Accepted: 8 October 2019

Published online: 17 October 2019 
accumbens (NAC) shell after treatment with $d$-Amphetamine using in vivo microdialysis [22]. The second aim of our study was to investigate functional neurobiological changes to the DA and DAT systems in rats with a history of alternating a standard chow with palatable diet. We quantified levels of tyrosine hydroxylase (TH) and DAT mRNA in the ventral tegmental area (VTA). We then employed the quantitative "no-net-flux" microdialysis procedure [23] to assess function of DAT and quantify baseline extracellular DA in vivo. We hypothesized that after a history of alternating standard chow with palatable diet, rats would display reduced reward system functioning and associated downregulation of the mesolimbic DA system.

\section{MATERIALS AND METHODS}

Supplemental material accompanies this paper

Subjects. Male Wistar rats $(n=134$; Charles River, Wilmington, MA) were single-housed in a 12-h reverse light cycle (lights off at 11:00 a.m.) in an AAALAC-approved humidity-controlled and temperature-controlled vivarium. Rats had access to corn-based chow (Harlan Teklad LM-485 Diet 7012; 44\% kcal carbohydrate, $5.8 \%$ fat, $19 \%$ protein, metabolizable energy $310 \mathrm{cal} / 100 \mathrm{~g}$; Harlan, Indianapolis, IN) and free access to water at all times with the exception of certain experimental test procedures. Procedures used in this study adhered to the National Institutes of Health Guide for the Care and Use of Laboratory Animals and were approved by Boston University Medical Campus Institutional Animal Care and Use Committee (approval no. 15174). Each experiment used an independent cohort of animals, with the exception of CPP, which took place prior to ICSS experiments.

Drugs. $d$-Amphetamine (Sigma Aldrich, St. Louis, MO) was dissolved in $0.9 \%$ sterile saline for intraperitoneal (i.p.) injections.

Ad libitum alternation of a standard chow with palatable diet. Ad libitum alternation of a standard chow with palatable diet was performed as described previously $[4,17,24,25]$ and continued for $\geq 5$ weeks preceding all experiments. Briefly, control rats were provided with free access to a chow diet 7 days a week (Chow/Chow) and experimental rats were provided with free access to chow for 5 days a week, followed by 2 days of free access to a palatable diet (Chow/Palatable). The "chow" diet was the above-described cornbased chow, and the palatable diet was a nutritionally complete, chocolate-flavored, high-sucrose $(50 \% \mathrm{kcal})$, AIN-76A-based diet that is comparable in macronutrient proportions and energy density to the chow diet $(45 \mathrm{mg}$, 5TUL: $66.7 \%$ kcal carbohydrate, $12.7 \%$ fat, $20.6 \%$ protein, metabolizable energy $344 \mathrm{kcal} / 100 \mathrm{~g}$; TestDiet, Richmond, IN). For brevity, the first 5 days (chow only) and the last 2 days (chow or palatable diet according to the experimental group) of each week are referred to in all experiments as $C$ and $P$ Phase, respectively. Diets were never concurrently available.

Locomotor activity. A locomotor activity assay was used to test sensitivity to the stimulating effects of $d$-Amphetamine. One day after food switch, rats (Chow/Chow, $n=12$; Chow/Palatable, $n=12$ ) were injected with saline or $d$-Amphetamine $(0.1,0.3$, and $1 \mathrm{mg} / \mathrm{kg}$, i.p.) using a counterbalanced, within-subject, Latin square design. All rats received each dose during both the $C$ and $P$ Phase, $>5$ days apart, and were compared against the Chow/Chow rats run on the same day. Locomotor response to $d$-Amphetamine is operationalized as the increase in locomotor activity as compared to locomotor activity under vehicle baseline conditions.

Intracranial self-stimulation (ICSS). An ICSS procedure was used to assess how $d$-Amphetamine potentiates brain stimulation reward (BSR) (i.e. lowers the BSR threshold), a measure of reward system functioning). BSR thresholds were determined using the rate-independent discrete-trial current intensity procedure
$[26,27]$. In each session, rats were required to lever press for electrical stimulation at varying current intensities. There were six total sets of trials (termed "columns") where current intensities were varied in either ascending or descending patterns. Within each column, rats performed five trials at each current intensity: $\geq 3$ out of 5 responses was considered a positive response at that intensity, while $\leq 2$ responses was considered a negative response. The threshold (i.e. current intensity where self-stimulation behavior ceases, defined as the midpoint between the last "positive response" intensity and the first "negative response" intensity) was determined for each column and averaged over the last four columns of each session. A decrease in the reward threshold reflects an increase in reward function as measured by ICSS $[26,28,29]$. On test days, rats were administered saline or $d$ Amphetamine $(0.1,0.3,1.0 \mathrm{mg} / \mathrm{kg}$, i.p.) immediately prior to the session start (one day post-diet switch), in a counterbalanced, within-subject, Latin square design (final group sizes: Chow/Chow, $n=7$; Chow/Palatable, $n=16$ ).

Conditioned place preference (CPP). A biased CPP procedure was used to test the rewarding effects of $d$-Amphetamine in diet alternated rats. Following 6 weeks of diet alternation, Chow/Palatable rats were maintained on either the chow food (C Phase group) or the palatable food ( $P$ Phase group) for the duration of CPP training/testing (7 days) and each group was run simultaneously with a Chow/Chow control group. During preconditioning, rats were allowed to freely explore the apparatus for $15 \mathrm{~min}$. On alternate days, rats (C Phase testing: Chow/Chow, $n=8$, Chow/Palatable: C Phase, $n=13$; P Phase testing: Chow/Chow, $n=6$, Chow/Palatable: $\mathrm{P}$ Phase, $n=12$ ) were given an injection of saline or $d$-Amphetamine $(1.0 \mathrm{mg} / \mathrm{kg}$, i.p.) and confined to one chamber for $25 \mathrm{~min}$, three pairings each $\quad(d$-Amphetamine $=$ unbiased chamber; saline $=$ biased chamber). The day after the last conditioning session, rats were tested following the same procedure as the preconditioning test. A CPP score was calculated (time in $d$-Amphetamine paired chamber-time in unpaired chamber $[30,31])$.

In vivo microdialysis. An in vivo microdialysis procedure was used to determine NAc shell DA efflux in response to $d$-Amphetamine. Rats were stereotaxically implanted with a unilateral, intracranial cannula in the NAc shell, as described previously [32-34]. In vivo microdialysis occurred in rats one day post-diet switch (either $C$ to $P$, or $\mathrm{P}$ to $\mathrm{C}$ Phase) for Chow/Palatable rats with Chow/Chow controls run simultaneously. Samples were collected at $10 \mathrm{~min}$ intervals at baseline (30 min), after saline injection (i.p.; $30 \mathrm{~min}$ ), and after injection with $d$-Amphetamine $(1 \mathrm{mg} / \mathrm{kg}$, i.p.; $2 \mathrm{~h})$. Samples were frozen and stored at $-80^{\circ} \mathrm{C}$ until high performance liquid chromatography (HPLC) analysis (final $n$ sizes: Chow/Chow, $n=6$; Chow/Palatable: C Phase, $n=8$; Chow/Palatable: P Phase, $n=9$ ).

Quantitative "no-net-flux" microdialysis. In order to determine baseline extracellular DA and DAT function, quantitative "no-netflux" microdialysis methods were used. Methods were identical to in vivo microdialysis methods described above, unless otherwise noted. Quantitative "no-net-flux" microdialysis was performed in rats (Chow/Chow, $n=8$; Chow/Palatable: C Phase, $n=7$; Chow/ Palatable: P Phase, $n=10$ ) one day post-diet switch (either $C$ to $\mathrm{P}$ or $\mathrm{P}$ to $($ Phase) for Chow/Palatable rats, with Chow/Chow controls run simultaneously. Varying concentrations of DA $(0,2.5,5$, and $10 \mathrm{nM})$ were added to the perfusate (1:3 mixture of aCSF and antioxidant, $\mathrm{pH}$ 6.8). A 90-min equilibration period was allowed after changing perfusate. Samples were collected at 10-min intervals for $30 \mathrm{~min}$. Loss or gain of DA into the perfusate was calculated [DA concentration in perfusate-DA concentration in collected sample] and plotted in a regression against DA concentration in perfusate. The slope (extraction fraction) and $x$-intercept were then calculated using linear regression for each animal. 

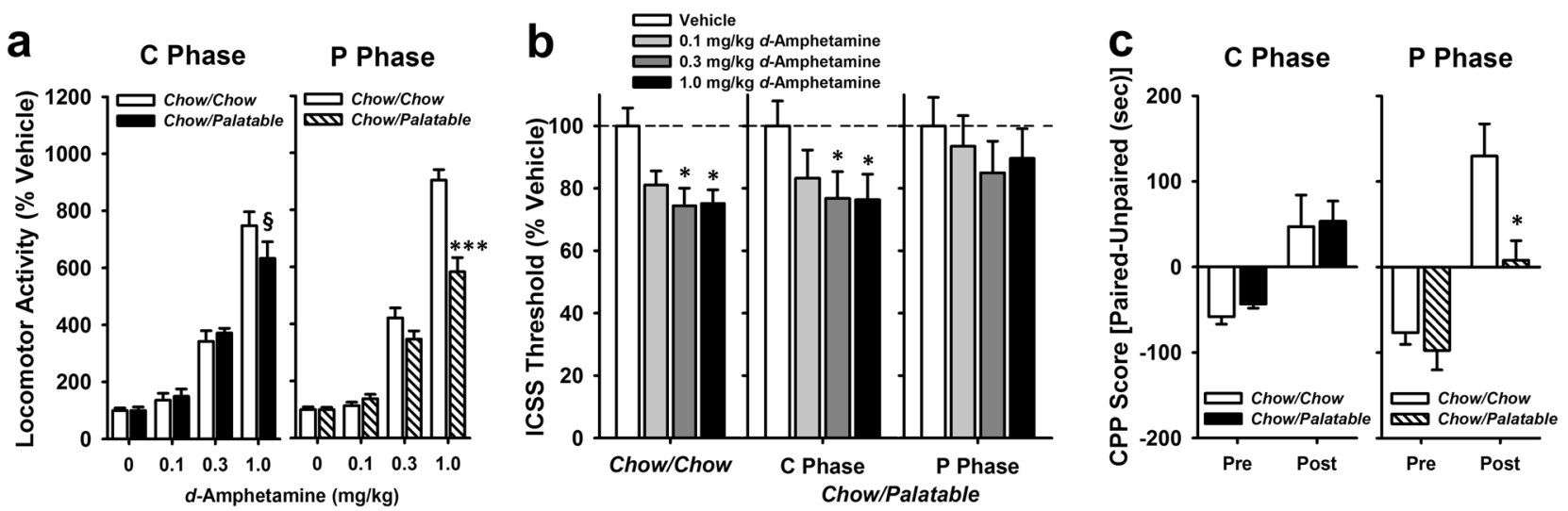

Fig. 1 Palatable diet alternated rats show a blunted response to $d$-Amphetamine when tested in the P Phase. Data are Mean $\pm \mathrm{SEM}$. a Locomotor activity after $d$-Amphetamine treatment (\% vehicle activity) $n=24$. ${ }^{* *} p<0.001$ vs. Chow/Chow group, $\S p=0.07$ vs. Chow/Chow group. b ICSS thresholds after $d$-Amphetamine treatment. $n=23 .{ }^{*} p<0.05$ compared to the Vehicle treatment within the same group. c Conditioned place preference to a $1 \mathrm{mg} / \mathrm{kg} d$-Amphetamine paired compartment. $n=39 .{ }^{*} p<0.05$ compared to the post test CPP score of the Chow/Chow group

High-performance liquid chromatography (HPLC). DA was measured by HPLC (HTEC-510, WE-3G electrochemical detector, Amuza Inc., San Diego, CA) [35, 36]. DA concentrations were determined by using standard curves with known amounts of DA in a range of $0.5-20 \mathrm{pg}$, with a detection limit of $0.02 \mathrm{pg}$. Retention times for DA were verified daily using standard solutions.

Histology. Rats that underwent in vivo microdialysis were sacrificed for brain histology placement at the culmination of the experiment.

Quantitative polymerase chain reaction $(q P C R)$. For the quantification of TH and DAT mRNA in the VTA, a separate, untested cohort of rats was sacrificed $24 \mathrm{~h}$ post-diet switch either from chow to palatable ( $P$ Phase, $n=8$ ) or from palatable to chow (C Phase, $n=9)$, along with Chow/Chow controls $(n=6)$. Procedures were performed as described previously [37]. Results were analyzed by second derivative methods and expressed in arbitrary units (normalized to the reference gene Cyclophilin A, Cyp; (see Supplemental Table 1 for primers and conditions).

Statistical analysis. Data from locomotor activity, ICSS, CPP, PCR, and quantitative no-net-flux (slope and x-intercept) experiments, as well as body weights, were analyzed with simple or factorial ANOVAs. To control for unspecified day effects, locomotor activity and CPP were analyzed against the simultaneously run control group. In vivo microdialysis data were analyzed using linear mixed effects modeling in order to account for missing at random data.

Pairwise post hoc comparisons were made using Newman-Keuls after significant ANOVAs. For mixed linear effects analysis, we used Bonferroni correction, a more conservative post hoc. Partial eta squared is reported as effect size for ANOVAs (main effects and interactions), while Cohen's $d$ was calculated according to Cohen [38] in the event of significant differences between groups as determined by post hoc analysis.

The software/graphic packages used were SigmaPlot 12.0 (Systat Software Inc., Chicago, IL), SPSS Statistics 24 (IBM Corp., Armonk, NY), and Statistica 7.0 (StatSoft, Tulsa, OK).

\section{RESULTS}

Chow/Palatable rats show blunted stimulatory response to $d$ Amphetamine: locomotor activity

During the P Phase, Chow/Palatable rats showed higher baseline locomotor activity compared to Chow/Chow rats (1388.5 \pm 128.9 vs. $2138.2 \pm 167.1$ beam breaks of Chow/Chow and Chow/Palatable rats tested during the $\mathrm{P}$ Phase; data not shown). An ANOVA of baseline locomotor activity revealed a main effect of Diet $\left(F(1,22)=8.69, p=0.01\right.$, partial $\left.\eta^{2}=0.28\right)$. Baseline locomotor activity was not different between groups during the $C$ Phase.

During the $\mathrm{P}$ Phase, Chow/Palatable rats displayed reduced locomotor response to $d$-Amphetamine compared to Chow/ Chow rats when tested with the highest dose $(1.0 \mathrm{mg} / \mathrm{kg}$; Fig. 1a). ANOVA of locomotor response to $d$-Amphetamine when tested in the $P$ Phase revealed main effects of Diet $(F(1,66)=$ 13.00, $p=0.002$, partial $\left.\eta^{2}=0.37\right)$ and Dose $(F(3,66)=286.27$, $p<0.0001$, partial $\left.\eta^{2}=0.93\right)$, as well as an interaction of Dose $x$ Diet $\left(F(3,66)=20.04, p<0.0001\right.$, partial $\left.\eta^{2}=0.48\right)$. Post-hoc analysis revealed differences between Chow/Chow and Chow/ Palatable at the $1 \mathrm{mg} / \mathrm{kg}$ dose ( $p=0.0001$, Cohen's $d=2.12)$. An ANOVA of rats' locomotor response to $d$-Amphetamine when tested in the $C$ Phase revealed no main effect of diet $(F(1,66)=$ $0.36, p=0.55$, partial $\left.\eta^{2}=0.02\right)$, but a main effect of Dose $(F$ $(3,66)=166.22, p<0.0001$, partial $\left.\eta^{2}=0.88\right)$ and a trend for a Dose $\times$ Diet interaction $\left(F(3,66)=2.47, p=0.07\right.$, partial $\eta^{2}=$ $0.10)$. Post hoc tests did not indicate significant differences between diet groups, but there was a trend for a difference between Chow/Palatable and Chow/Chow rats at the $1 \mathrm{mg} / \mathrm{kg}$ dose when tested in the $C$ phase $(p=0.07$, Cohen's $d=0.62$ ). Body weights were not different between groups in $C$ Phase $\left(\mathrm{t}(1,22)=0.55, p=0.59\right.$, partial $\left.\eta^{2}=0.01\right)$ or $\mathrm{P}$ Phase $(\mathrm{t}(1,22)=$ $-0.01, p=0.99$, partial $\eta^{2}=0.00$; data not shown).

Chow/Palatable rats are less sensitive to $d$-Amphetamine enhancement of BSR: ICSS

BSR thresholds of Chow/Palatable rats were insensitive to pretreatment with $d$-Amphetamine during the $\mathrm{P}$ Phase $\left(0.3<p^{\prime} \mathrm{s}<0.8\right.$; Fig. 1b), while $d$-Amphetamine pre-treatment decreased BSR thresholds from baseline in both Chow/Chow rats and Chow/ Palatable rats during the $C$ Phase $\left(p^{\prime} s<0.05\right)$. An ANOVA on BSR threshold after pre-treatment with $d$-Amphetamine or vehicle indicated a main effect of Dose $(F(3,108)=10.71, p<0.0001$, partial $\left.\eta^{2}=0.23\right)$, though there was no main effect of Diet Group $\left(F(2,108)=0.37, p=0.70\right.$, partial $\left.\eta^{2}=0.02\right)$, nor a Diet Group $\times$ Dose interaction $\left(F(6,108)=0.57, p=0.75\right.$, partial $\left.\eta^{2}=0.03\right)$. Body weights were not different between groups $(\mathrm{F}(1,22)=1.22, p=$ 0.73 , partial $\eta^{2}=0.01$; data not shown).

Chow/Palatable rats are less sensitive to the rewarding effects of $d$ Amphetamine: CPP

When conditioned and tested in the P Phase, Chow/Palatable rats showed a lower preference for the $d$-Amphetamine paired 
chamber compared to Chow/Chow rats in the post-conditioning test (Fig. 1c; $p=0.03$, Cohen's $d=1.46$ ). ANOVA of CPP scores of rats conditioned and tested in the $P$ Phase revealed main effects of Day $\left(F(1,16)=32.65, p<0.0001\right.$, partial $\left.\eta^{2}=0.67\right)$ and Diet Group $\left(F(1,16)=7.17, p=0.02\right.$, partial $\left.\eta^{2}=0.31\right)$, as well as a trend for an interaction of Diet Group $\times$ Day $(F(1,16)=3.43, p=$ 0.08 , partial $\left.\eta^{2}=0.18\right)$. When conditioned and tested in the C Phase, Chow/Palatable and Chow/Chow rats showed equivalent increased preference for the $d$-Amphetamine paired chamber. ANOVA revealed main effects of Day $(F(1,19)=26.1$, $p<0.0001$, partial $\left.\eta^{2}=0.58\right)$, but not Diet Group $(F(1,19)=0.22$, $p=0.65$, partial $\left.\eta^{2}=0.01\right)$, and the interaction of Diet Group $\times$ Day was not significant $\left(\mathrm{F}(1,19)=0.05, p=0.83\right.$, partial $\eta^{2}=$ 0.002). CPP scores on the pre-test day were not different between diet groups. Body weights were not different between groups tested in the $C$ Phase $(F(1,19)=0.94, p=0.36$, partial $\left.\eta^{2}=0.05\right)$ or $\mathrm{P}$ Phase $\left(\mathrm{t}(1,16)=0.44, p=0.66\right.$, partial $\left.\eta^{2}=0.01\right)$; data not shown).

d-Amphetamine-induced DA efflux is impaired in Chow/Palatable rats

DA efflux in response to $d$-Amphetamine was reduced in Chow/ Palatable rats ( $\mathrm{C}$ and $\mathrm{P}$ Phase) compared to Chow/Chow rats (20 min post-injection; Fig. $2, p^{\prime} s<0.05$, Cohen's $d=0.80$ and 1.42 for Chow/Palatable: C Phase and Chow/Palatable: $\mathrm{P}$ Phase, respectively). Chow/Palatable: $\mathrm{P}$ Phase rats continued to show lower DA compared to Chow/Chow $30 \mathrm{~min}$ after $d$-Amphetamine injection $(p=0.003$, Cohen's $d=1.25)$. Chow/Palatable: C Phase rats also tended to have lower DA compared to Chow/Chow rats at $30 \mathrm{~min}$ post-injection ( $p=0.07$, Cohen's $d=0.75$ ). An ANOVA of DA efflux following $d$-Amphetamine revealed a trend for a main effect of Diet Group $(F(2,29.55)=3.12, p=0.06)$, a main effect of Time ( $F$ $(11,173.75)=31.94, \quad p<0.0001)$, and an interaction of Diet Group $\times$ Time $(F(22,173.81)=2.02, p=0.01)$. Body weights were not different between groups $(\mathrm{F}(1,22)=0.00, p=0.99$, partial $\eta^{2}=0.00 ;$ data not shown).

DAT mRNA is increased in Chow/Palatable rats during the $P$ Phase Chow/Palatable rats during the $P$ Phase had increased DAT mRNA expression compared to Chow/Chow rats $(p=0.03$, Cohen's $d=$ $-0.96)$ and Chow/Palatable rats during the $C$ Phase $(p=0.01$, Cohen's $d=-1.59$; Fig. $3 a, b)$. ANOVA revealed a main effect of Diet Group on DAT mRNA in the VTA $(F(2,20)=5.74, p=0.01$, partial $\left.\eta^{2}=0.37\right)$. There were no differences in DAT mRNA between Chow/Palatable: $C$ Phase and Chow/Chow rats $(p=0.42$, Cohen's $d=0.60)$. TH mRNA was not affected by Diet Group $\left(F(2,20)=0.013, p=0.88\right.$, partial $\left.\eta^{2}=0.01\right)$. Body weights were not different between groups $(F(1,22)=2.34, p=0.12$, partial $\eta^{2}=0.19 ;$ data not shown).

Baseline DA and DAT function is reduced in Chow/Palatable rats Chow/Palatable rats in both $C$ Phase and $\mathrm{P}$ Phase had lower baseline DA compared to Chow/Chow rats ( $p$ 's $<0.05$; Cohen's $d=$ 1.13 and 0.95 for Chow/Palatable: C Phase and Chow/Palatable: $P$ Phase, respectively; Fig. 4a). An ANOVA revealed significant main effects of Diet Group on baseline extracellular DA $(F(2,22)=3.67$, $p=0.04$, partial $\eta^{2}=0.25$ ).

Clearance of DA was lower in Chow/Palatable rats: $C$ Phase $(p=$ 0.03, Cohen's $d=1.13$ ), and tended to be lower in Chow/Palatable rats: $\mathrm{P}$ Phase rats compared to Chow/Chow rats $(p=0.06$, Cohen's $d=1.10$; Fig. 4b). ANOVA revealed a main effect of Diet Group on the clearance rate of DA by DAT, indicated by the slope (extraction fraction) of regression lines $\left(F(2,22)=3.87, p=0.04\right.$, partial $\eta^{2}=$ 0.26 ; plots of regression lines can be seen in Fig. $4 \mathrm{c}$; see Fig. $5 \mathrm{a}-\mathrm{c}$ for cannula placement for all microdialysis experiments). Body weights were not different between groups $(F(1,24)=1.54, p=$ 0.24 , partial $\eta^{2}=0.12$; data not shown).

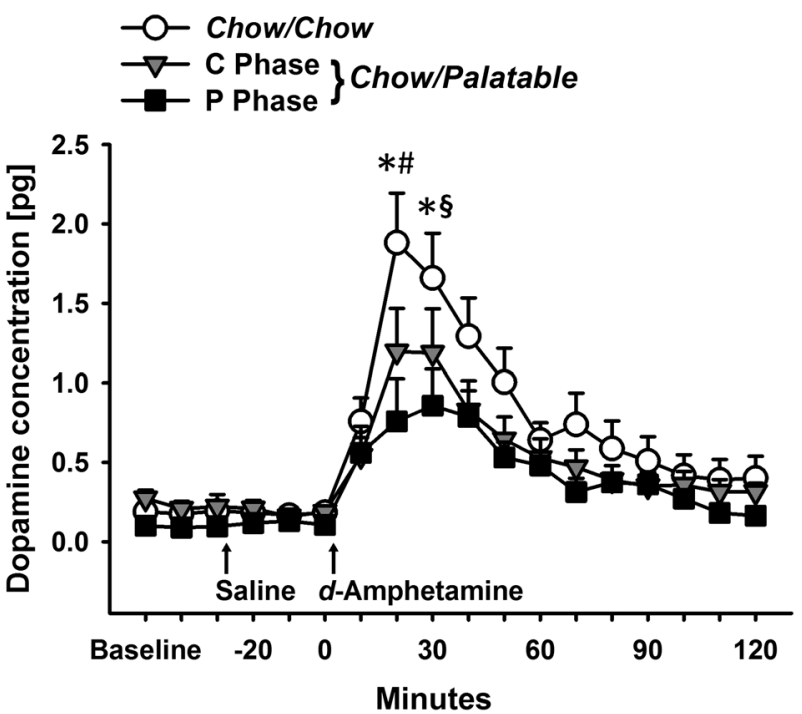

Fig. 2 Reduced DA efflux following treatment with $d$-Amphetamine $(1 \mathrm{mg} / \mathrm{kg})$ in Chow/Palatable rats. Data are Mean \pm SEM. $n=23 .{ }^{*} p<$ 0.05 Chow/Palatable: P Phase group vs. Chow/Chow group, \# $p<0.05$ and $\S p=0.07$ Chow/Palatable: C Phase group vs. Chow/Chow group

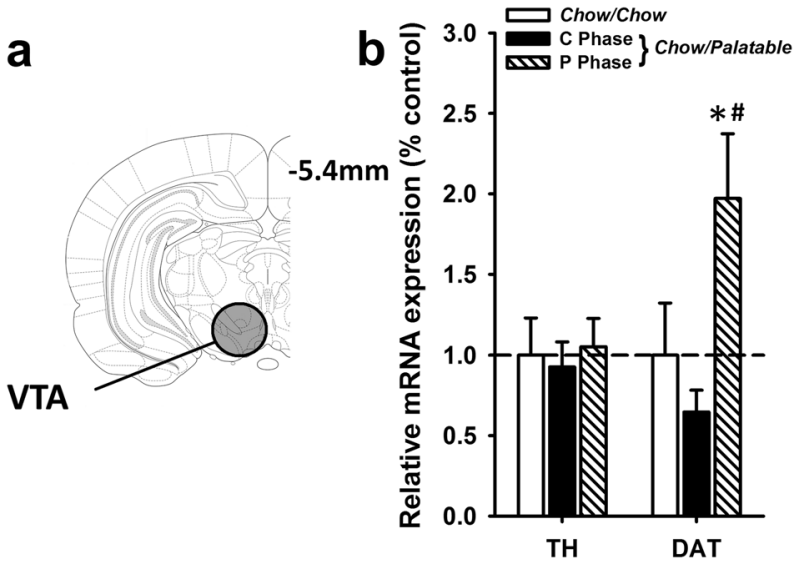

Fig. 3 DAT mRNA is increased in Chow/Palatable rats in the $P$ Phase. a Schematic of VTA section harvested for qPCR analysis. b mRNA expression (normalized to Cyp) of TH and DAT is expressed relative to Chow/Chow group levels. Data are Mean \pm SEM. $n=23 .{ }^{*} p<0.05$ vs. Chow/Chow group; $\# p<0.05$ vs. Chow/Palatable: C Phase group

\section{DISCUSSION}

Reduced sensitivity to $d$-Amphetamine

These results provide experimental evidence that alternation of a standard chow with palatable diet, a validated animal model of compulsive eating behavior $[4,17,24,39]$, reduces sensitivity to $d$ Amphetamine, suggestive of a hypofunctional reward system. Specifically, Chow/Palatable rats displayed blunted $d$-Amphetamine-heightened locomotor activity, no potentiation of BSR threshold by $d$-Amphetamine, and decreased place preference for $d$-Amphetamine, when access to highly palatable diet was renewed.

Preclinical studies investigating the effects of overconsumption of high-fat and/or high-sucrose diets on sensitivity to psychostimulants have shown mixed results in part due to the heterogeneity of the experimental conditions (diet formulations, access types, stimulant-type, etc). For example, in a study by Fordahl et al. [40], mice with limited access to a high-fat diet showed increased locomotor activity (\% of baseline) after 

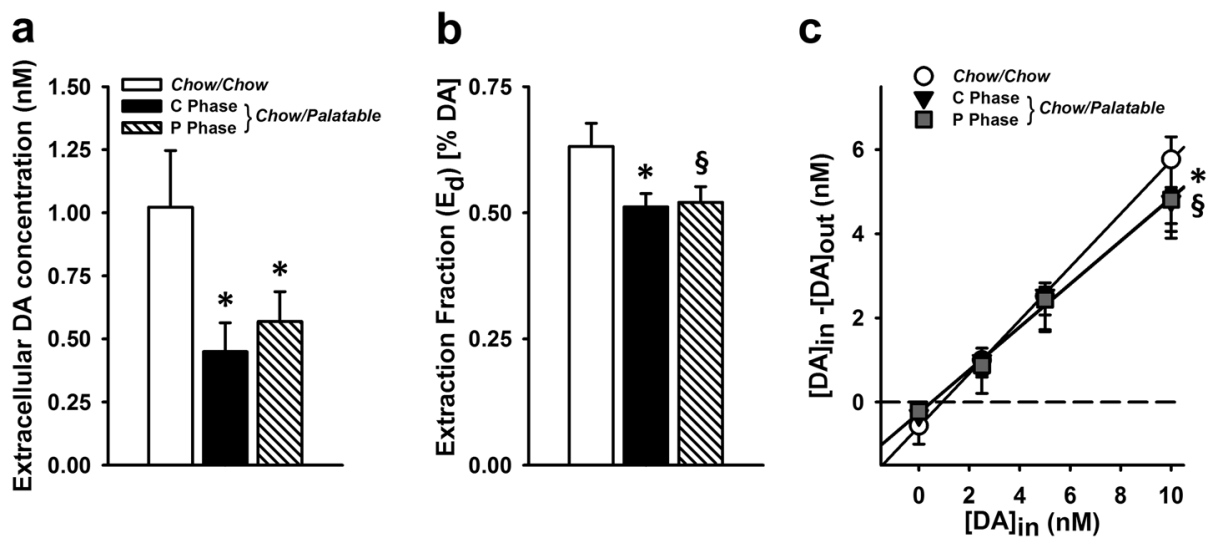

Fig. 4 Quantitative 'no-net-flux' microdialysis reveals DA system neuroadapatations in Chow/Palatable rats. Data are Mean \pm SEM. a Extracellular DA concentration ${ }^{*} p<0.05$ vs. Chow/Chow group. b Extraction fraction ( $\mathrm{E}_{\mathrm{d}} ;$ slope) ${ }^{*} p<0.05$ vs. Chow/Chow group, $\S p=0.06$ vs. Chow/Chow group. c plotted regression analysis. ${ }^{*} p<0.05$ Chow/Palatable: C Phase group vs. Chow/Chow group; $\S p=0.06$ Chow/Palatable: P Phase group vs. Chow/Chow group. $n=25$ for all panels
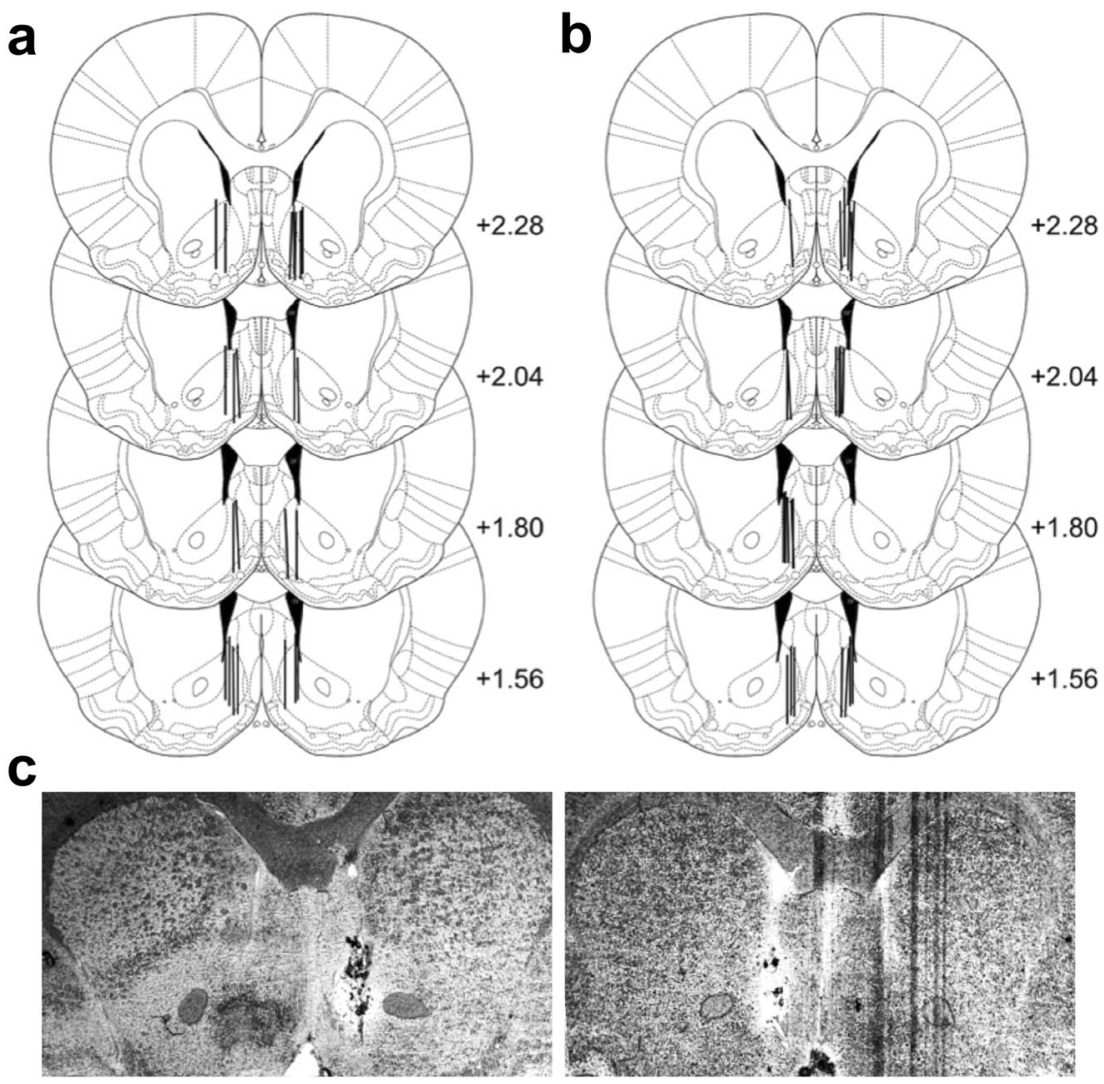

Fig. 5 Cannula placement for microdialysis experiments; a in vivo microdialysis after $d$-Amphetamine and b quantitative "no-net-flux" microdialysis. c Representative photomicrographs of NAc shell placement

treatment with $d$-Amphetamine $(3.0 \mathrm{mg} / \mathrm{kg}$ after receiving $0.5 \mathrm{mg} /$ $\mathrm{kg}$ the previous day) compared to control mice. However, in this study, baseline activity was actually lower in the high-fat diet fed mice, and therefore the total locomotor activity (i.e. not expressed as a \% of baseline) that was increased by the first low dose $(0.5 \mathrm{mg} / \mathrm{kg})$ of $d$-Amphetamine, prior to the start of the sensitization protocol, was likely lower compared to controls [40], which would be more in line with results from the present study. However, inconsistent results between the existing and the present study are not surprising and likely due to differences in the administration protocol (spacing of injections, doses used), species (mice vs. rats), and diets (high-fat vs. high sucrose) [40]. In the current study, we observed lower $d$-Amphetamine heightened locomotor activity in Chow/Palatable rats compared to controls. Though we did not directly compare across phases due to nonspecific day effects in Chow/Chow control animals, our data did not suggest any differential effects of diet phase on locomotor response in Chow/Palatable rats; rather they, if anything, would indicate differences in magnitude.

We confirmed that the ad libitum alternation of a standard chow with palatable diet does not alter brain reward threshold per se (i.e. no differences in baseline BSR in discrete-trial current intensity procedures, including no differences between $C$ and $P$ Phase) [17]. Reward deficits in the form of increased brain 
stimulation thresholds have been observed in rats following the development of high-fat diet induced obesity [41]. The fact that we did not observe differences in baseline BSR threshold in our model may be due to a lack of an overweight phenotype, as rats with intermittent access to a palatable diet maintained equivalent body weight compared to controls. In the same study, and consistent with our results, Johnson and Kenny [41] observed that rats with intermittent restricted daily access to a high-sugar/highfat diet did not become obese, and did not display changes in baseline BSR thresholds compared to controls. Nonetheless, using a more sensitive probing of reward system function via assessment of $d$-Amphetamine's potentiating effects on BSR threshold [42], here we observed decreased reward system function in Chow/Palatable rats re-exposed to the highly palatable diet. Thus, similarly to what was observed with the locomotor-stimulating effects of $d$-Amphetamine, rats re-exposed to the palatable diet were less sensitive to the potentiating effects of $d$-Amphetamine on brain reward function.

Our results of blunted place conditioning to $d$-Amphetamine in Chow/Palatable rats suggest reduced sensitivity to the primary rewarding effects of $d$-Amphetamine following a history of intermittent palatable diet acess. Accordingly, both rats fed a high-fat diet ad libitum and allowed to develop obesity or rats fed a restricted amount of high-fat diet to limit weight gain did not show CPP for $d$-Amphetamine (1.0 mg/kg) [13]. In contrast to our findings, rats allowed continuous access to a sucrose solution in addition to laboratory chow showed a higher CPP score for a low dose of $d$-Amphetamine $(0.33 \mathrm{mg} / \mathrm{kg})$ compared to chow rats [43], suggesting increased sensitivity. The discrepancy between this study and ours is most likely due to differences in pattern of palatable food access (intermittent vs. continuous); indeed, different intake patterns often produce distinct behavioral and neurobiological outcomes [44, 45], where intermittent access more reliably produce maladaptive binge- and compulsive-like intake compared to continuous access. The importance of the intermittency of the palatable diet access in the development/expression of reward deficits is not fully understood, in large part due to the confounds of increased body weight that occurs with continuous access to a palatable diet. There is difficulty in disentangling schedule of access effects (for example, here through the inclusion of a continuously palatable diet fed group) in food studies without also introducing a confounding factor of obesity or increased body weight. Furthermore, running a continuous ad libitum palatable food group would necessarily leave a mismatch in a different control variable (e.g. days of access allowed or quantity (kcal) of palatable food eaten). Controlling for intermittency inevitably results in disruptions of amount of palatable food eaten between groups, not allowing for full control of all interpretative factors of interest.

Interestingly, blunted behavioral sensitivity to $d$-Amphetamine was observed during the $P$ Phase, but not the $C$ Phase. While it has been hypothesized that compulsive eating to relieve a negative emotional state can be driven by both elevated stress and diminished reward capacity $[1,5]$, it was previously unknown whether these factors are related, or manifest independently. Our previous studies show that withdrawal from palatable food during the $C$ Phase produces anxiety- and depressive-like behavior [17, 46, 47], which indicates elevated stress. However, the present experiments demonstrate diminished reward sensitivity outside of the withdrawal state, when palatable food was available (i.e. P Phase). This temporal dissociation suggests that the impact of stress and reward deficits on compulsive eating may be mediated by independent mechanisms. It is currently unknown if this is shared with drug addiction, as testing reward deficits in animal models of drug dependence is often confounded by drug intoxication. Investigations into reward deficits in the context of food may therefore represent a unique opportunity to uncover distinctions between these dual components in an addiction-like disorder.

Functional changes to DA system in Chow/Palatable rats Our results show that DA efflux in the NAc shell following an injection of $d$-Amphetamine was significantly reduced in Chow/ Palatable rats as compared to controls. This neurochemical evidence of impaired DA system functioning in response to $d$ Amphetamine may, therefore, represent the neural mechanism of the insensitivity to $d$-Amphetamine we observed.

Furthermore, using qPCR, we observed increased DAT mRNA in Chow/Palatable rats during the $P$ Phase. To follow up on this finding, we investigated DAT function using quantitative "no-netflux" microdialysis methods, and observed reduced DA clearance (i.e. lower DAT function) in Chow/Palatable rats. This method also allowed for the quantification of extracellular DA at baseline, which was also reduced in Chow/Palatable rats. Taken together, these experiments show multiple functional neuroadaptations to the DA system in Chow/Palatable rats, manifesting as an overall downregulation of the mesolimbic DA system.

In accordance with these findings, a study of female rats made obese through continuous access to a palatable diet also exhibited reduced baseline DA, as well as lower electrically evoked and $d$ Amphetamine induced DA $[10,11]$. In our experiments, the reduced DA efflux following $d$-Amphetamine observed in Chow/ Palatable rats is most likely mediated via reduced baseline extracellular DA and lower DAT function. As DAT is the main substrate of $d$-Amphetamine, dysfunctional DAT reduces the efficacy of $d$-Amphetamine [48]. This hypothesized mechanism is confirmed by the results obtained from the "no-net-flux" experiment, where we observed reduced baseline extracellular DA and lower DAT function in Chow/Palatable rats. As "no-net-flux" methods assess the functional status of DAT in vivo, the reduced reward sensitivity to $d$-Amphetamine observed in Chow/Palatable animals could reflect either lower number of functional membrane-bound DAT or less-efficient membrane-bound DAT. Furthermore, our observation of increased DAT mRNA in Chow/ Palatable rats suggests that reductions in DAT function (i.e. reduced number and/or efficiency of DAT) may be responsible for a compensatory increase in mRNA that is observable during the $P$ Phase. Other preclinical studies have also observed increases in DAT mRNA in obesity-prone [49], or sucrose bingeing [50] rats. In support of our findings, a study looking at effects of intermittent high-fat diet access also found reductions in DAT function, observed using fast-scan cyclic voltammetry [40].

We observed that Chow/Palatable rats in the $C$ Phase showed evidence of DA system dysfunction, but did not display observable differences in behavioral response to $d$-Amphetamine. Specifically, Chow/Palatable rats showed lower DA efflux after $d$-Amphetamine treatment, as well as reduced baseline extracellular DA and DAT function, but when tested in the C Phase, behavioral measures of $d$-Amphetamine sensitivity were spared. A speculative explanation for these findings may be that DA is reduced in both $C$ and $P$ Phase, but the stores of DA are further depleted by recent palatable food intake in $P$ Phase, resulting in lower overall DA efflux in response to $d$-Amphetamine and observable differences in behavioral sensitivity to $d$-Amphetamine. Therefore, the observed reward sensitivity deficits are due to an interaction between a history of alternation of a standard chow with palatable diet and present feeding state. An alternative explanation for the discrepancy between DA efflux and behavioral response is that compensatory changes to postsynaptic DA receptor expression counteract the observed changes to DA release-effectively masking the underlying neurochemical dysfunction. Therefore, future studies should examine additional DA signaling components that might undergo changes throughout feeding cycles to hone our understanding of DA system neuroadaptations that occur in compulsive eating. 


\section{CONCLUSIONS}

A neurobiological underpinning of compulsive eating behavior is overeating to relieve a negative emotional state of which the neural mechanisms are (1) an increase in negative emotional states upon withdrawal and (2) a downregulation of brain reward function. We have previously characterized the emergence of a negative emotional state in palatable diet alternated rats $[4,17]$. The experiments presented here provide evidence for a concurrent reduction in brain reward function, thus suggesting that intermittent overeating of palatable food causes reward deficits, perhaps through neuroadaptations to mesolimbic DA, which may then in turn contribute to the perpetuation of compulsive eating behavior.

\section{FUNDING AND DISCLOSURE}

This work was supported by grants DA044664 (CM), DA030425 (PC), AA026051 (PC), AA025038 (VS), AA024439 (VS), DA031734 (KM), and AA013983 (KM) from NIDA and NIAAA, by the Peter Paul Career Development Professorship (PC), the McManus Charitable Trust (VS), the Burroughs Wellcome Fund through the Transformative Training Program in Addiction Sciences (CM). Its contents are solely the responsibility of the authors and do not necessarily represent the official views of the National Institutes of Health. The authors declare no conflict of interest.

\section{ADDITIONAL INFORMATION}

Supplementary Information accompanies this paper at (https://doi.org/10.1038/ s41386-019-0550-1).

Publisher's note Springer Nature remains neutral with regard to jurisdictional claims in published maps and institutional affiliations.

\section{REFERENCES}

1. Moore CF, Sabino V, Koob GF, Cottone P. Pathological overeating: emerging evidence for a compulsivity construct. Neuropsychopharmacology. 2017;42:1375-89.

2. Koob GF. Drug addiction: the yin and yang of hedonic homeostasis. Neuron. 1996;16:893-6.

3. Parylak SL, Koob GF, Zorrilla EP. The dark side of food addiction. Physiol Behav. 2011;104:149-56

4. Cottone P, Sabino V, Roberto M, Bajo M, Pockros L, Frihauf JB, et al. CRF system recruitment mediates dark side of compulsive eating. Proc Natl Acad Sci USA. 2009;106:20016-20.

5. Koob GF. Addiction is a reward deficit and stress surfeit disorder. Front Psychiatry. 2013;4:72.

6. Moore CF, Sabino V, Koob GF, Cottone P. Neuroscience of compulsive eating behavior. Front Neurosci. 2017;11:469.

7. Melis $M$, Spiga S, Diana M. The dopamine hypothesis of drug addiction: hypodopaminergic state. Int Rev Neurobiol. 2005;63:101-54.

8. O'Connor RM, Kenny PJ. Role of striatal dopamine signaling in compulsive eating associated with obesity. Curr Opin Behav Sci. 2016;9:152-7.

9. Moore, CF, Panciera, Jl, Sabino, V, and Cottone, P. Neuropharmacology of compulsive eating. Philos Trans $R$ Soc Lond B Biol Sci. 2018;373. https://doi.org/ 10.1098/rstb.2017.0024.

10. Geiger BM, Haburcak M, Avena NM, Moyer MC, Hoebel BG, Pothos EN. Deficits of mesolimbic dopamine neurotransmission in rat dietary obesity. Neuroscience. 2009;159:1193-9.

11. Wang GJ, Volkow ND, Logan J, Pappas NR, Wong CT, Zhu W, et al. Brain dopamine and obesity. Lancet. 2001;357:354-7.

12. Hajnal A, Norgren R. Repeated access to sucrose augments dopamine turnover in the nucleus accumbens. Neuroreport. 2002;13:2213-6.

13. Davis JF, Tracy AL, Schurdak JD, Tschop MH, Lipton JW, Clegg DJ, et al. Exposure to elevated levels of dietary fat attenuates psychostimulant reward and mesolimbic dopamine turnover in the rat. Behav Neurosci. 2008;122:1257-63.

14. South T, Huang XF. High-fat diet exposure increases dopamine D2 receptor and decreases dopamine transporter receptor binding density in the nucleus accumbens and caudate putamen of mice. Neurochem Res. 2008;33:598-605.

15. Dore R, Valenza M, Wang X, Rice KC, Sabino V, Cottone P. The inverse agonist of CB1 receptor SR141716 blocks compulsive eating of palatable food. Addict Biol. 2014;19:849-61.
16. Moore CF, Cheng JE, Sabino V, Cottone P. Modeling and testing compulsive eating behaviors in animals. In: Cottone $\mathrm{P}$, Sabino V, Moore CF, Koob GF, editors. Compulsive eating behavior and food addiction. New York, NY: Academic Press; 2019. p.359-388.

17. lemolo A, Valenza M, Tozier L, Knapp CM, Kornetsky C, Steardo L, et al. Withdrawal from chronic, intermittent access to a highly palatable food induces depressive-like behavior in compulsive eating rats. Behav Pharm. 2012;23: 593-602.

18. Blasio A, Rice KC, Sabino V, Cottone P. Characterization of a shortened model of diet alternation in female rats: effects of the $C B 1$ receptor antagonist rimonabant on food intake and anxiety-like behavior. Behav Pharm. 2014;25:609-17.

19. Fray PJ, Sahakian BJ, Robbins TW, Koob GF, Iversen SD. An observational method for quantifying the behavioural effects of dopamine agonists: contrasting effects of $d$-amphetamine and apomorphine. Psychopharmacol (Berl). 1980;69:253-9.

20. Kornetsky C, Esposito RU. Euphorigenic drugs: effects on the reward pathways of the brain. Fed Proc. 1979;38:2473-6.

21. Mucha RF, Iversen SD. Reinforcing properties of morphine and naloxone revealed by conditioned place preferences: a procedural examination. Psychopharmacol (Berl). 1984;82:241-7.

22. Ungerstedt U. Microdialysis-principles and applications for studies in animals and man. J Intern Med. 1991;230:365-73.

23. Parsons LH, Justice JB Jr. Extracellular concentration and in vivo recovery of dopamine in the nucleus accumbens using microdialysis. J Neurochem. 1992;58: 212-8.

24. Blasio A, lemolo A, Sabino V, Petrosino S, Steardo L, Rice KC, et al. Rimonabant precipitates anxiety in rats withdrawn from palatable food: role of the central amygdala. Neuropsychopharmacology. 2013;38:2498-507.

25. Cottone P, Sabino V, Steardo L, Zorrilla EP. Intermittent access to preferred food reduces the reinforcing efficacy of chow in rats. Am J Physiol Regul Integr Comp Physiol. 2008;295:R1066-76.

26. Markou A, Koob GF. Construct validity of a self-stimulation threshold paradigm: effects of reward and performance manipulations. Physiol Behav. 1992;51:111-9.

27. Esposito R, Kornetsky C. Morphine lowering of self-stimulation thresholds: lack of tolerance with long-term administration. Science. 1977;195:189-91.

28. Der-Avakian A, Markou A. The neurobiology of anhedonia and other rewardrelated deficits. Trends Neurosci. 2012;35:68-77.

29. Der-Avakian A, Mazei-Robison MS, Kesby JP, Nestler EJ, Markou A. Enduring deficits in brain reward function after chronic social defeat in rats: susceptibility, resilience, and antidepressant response. Biol Psychiatry. 2014;76:542-9.

30. Velazquez-Sanchez C, Santos JW, Smith KL, Ferragud A, Sabino V, Cottone P. Seeking behavior, place conditioning, and resistance to conditioned suppression of feeding in rats intermittently exposed to palatable food. Behav Neurosci. 2015;129:219-24.

31. Wang N, Su P, Zhang Y, Lu J, Xing B, Kang K, et al. Protein kinase D1-dependent phosphorylation of dopamine D1 receptor regulates cocaine-induced behavioral responses. Neuropsychopharmacology. 2014;39:1290-301.

32. Dore $R$, lemolo $A$, Smith $K L$, Wang $X$, Cottone $P$, Sabino V. CRF mediates the anxiogenic and anti-rewarding, but not the anorectic effects of PACAP. Neuropsychopharmacology. 2013;38:2160-9.

33. lemolo A, Ferragud A, Cottone P, Sabino V. Pituitary adenylate cyclase-activating peptide in the central amygdala causes anorexia and body weight loss via the melanocortin and the TrkB systems. Neuropsychopharmacology. https://doi.org/ 10.1038/npp.2015.34.

34. Smith KL, Rao RR, Velazquez-Sanchez C, Valenza M, Giuliano C, Everitt BJ, et al. The uncompetitive $\mathrm{N}$-methyl-D-aspartate antagonist memantine reduces bingelike eating, food-seeking behavior, and compulsive eating: role of the nucleus accumbens shell. Neuropsychopharmacology. 2015;40:1163-71.

35. Boyson CO, Holly EN, Shimamoto A, Albrechet-Souza L, Weiner LA, DeBold JF, et al. Social stress and CRF-dopamine interactions in the VTA: role in long-term escalation of cocaine self-administration. J Neurosci. 2014;34:6659-67.

36. Miczek KA, Nikulina EM, Shimamoto A, Covington HE 3rd. Escalated or suppressed cocaine reward, tegmental BDNF, and accumbal dopamine caused by episodic versus continuous social stress in rats. J Neurosci. 2011;31: 9848-57

37. Sabino V, Cottone $P$, Zhao $Y$, lyer MR, Steardo L Jr., Steardo L, et al. The sigmareceptor antagonist BD-1063 decreases ethanol intake and reinforcement in animal models of excessive drinking. Neuropsychopharmacology. 2009;34:1482-93.

38. Cohen J. Statistical power analysis for the behavioral sciences. Hillsdale, N.J: L. Erlbaum Associates; 1988.

39. lemolo A, Blasio A,St, Cyr SA, Jiang F, Rice KC, Sabino V, et al. CRF-CRF1 receptor system in the central and basolateral nuclei of the amygdala differentially mediates excessive eating of palatable food. Neuropsychopharmacology. 2013; 38:2456-66. 
Reward sensitivity deficits in a rat model of compulsive eating behavior CF Moore et al.

596

40. Fordahl SC, Locke JL, Jones SR. High fat diet augments amphetamine sensitization in mice: Role of feeding pattern, obesity, and dopamine terminal changes. Neuropharmacology. 2016;109:170-82.

41. Johnson PM, Kenny PJ. Dopamine D2 receptors in addiction-like reward dysfunction and compulsive eating in obese rats. Nat Neurosci. 2010;13: 635-41.

42. Valenza M, Steardo L, Cottone $P$, Sabino V. Diet-induced obesity and dietresistant rats: differences in the rewarding and anorectic effects of $D$ amphetamine. Psychopharmacol (Berl). 2015;232:3215-26.

43. Vitale MA, Chen D, Kanarek RB. Chronic access to a sucrose solution enhances the development of conditioned place preferences for fentanyl and amphetamine in male Long-Evans rats. Pharm Biochem Behav. 2003;74:529-39.

44. Corwin RL. Bingeing rats: a model of intermittent excessive behavior? Appetite. 2006;46:11-15.

45. Furlong TM, Jayaweera HK, Balleine BW, Corbit LH. Binge-like consumption of a palatable food accelerates habitual control of behavior and is dependent on activation of the dorsolateral striatum. J Neurosci. 2014;34:5012-22.
46. Cottone P, Sabino V, Steardo L, Zorrilla EP. Opioid-dependent anticipatory negative contrast and binge-like eating in rats with limited access to highly preferred food. Neuropsychopharmacology. 2008;33:524-35.

47. Cottone P, Sabino V, Steardo L, Zorrilla EP. Consummatory, anxiety-related and metabolic adaptations in female rats with alternating access to preferred food. Psychoneuroendocrinology. 2009;34:38-49.

48. Vengeliene $V$, Bespalov $A$, Rossmanith $M$, Horschitz $S$, Berger $S$, Relo $A L$, et al. Towards trans-diagnostic mechanisms in psychiatry: neurobehavioral profile of rats with a loss-of-function point mutation in the dopamine transporter gene. Dis Model Mech. 2017;10:451-61.

49. Alsio J, Olszewski PK, Norback AH, Gunnarsson ZE, Levine AS, Pickering $C$, et al. Dopamine D1 receptor gene expression decreases in the nucleus accumbens upon long-term exposure to palatable food and differs depending on dietinduced obesity phenotype in rats. Neuroscience. 2010;171:779-87.

50. Bello NT, Sweigart KL, Lakoski JM, Norgren R, Hajnal A. Restricted feeding with scheduled sucrose access results in an upregulation of the rat dopamine transporter. Am J Physiol Regul Integr Comp Physiol. 2003;284:R1260-8. 